\title{
The Age Problem and Growing of Structures for Open System Cosmology
}

\author{
M. de Campos and N.A. Tomimura \\ Instituto de Física \\ Universidade Federal Fluminense \\ Av. Gal. Milton Tavares de Souza $s / n^{\circ}$. \\ 24210-340, Niterói, RJ, Brasil
}

Received on 13 November, 2000

\begin{abstract}
A new equation for the density contrast is derived in the framework of reexamined Newtonian cosmology if we take into account adiabatic matter creation in the universe. The age of the universe and the reach of non linear regime of the density contrast are usually treated separately in the literature and this may lead to controversial conclusions regarding the most adequate scenario to describe the universe. We relate the age of the universe and the growing mode of the density contrast by introducing a variable $\xi$ that relates both of them, so that both aspects are treated simultaneously. We apply this procedure to the Friedmann type model where the source of particle production is $\Psi=3 n \beta H$.
\end{abstract}

\section{Introduction}

In 1934, E.A.Milne analyzed the expansion of the universe using elementary Newtonian theory [1]. In the same year W.H.Mc Crea and E.A.Milne demonstrated the identity between the governing differential equations for relativistic and Newtonian cosmology, for null pressure [2]. Three decades after, E.R.Harrison [3] extended Newtonian cosmology to include pressure, generalizing Mac Crea and Milne's approach for dust matter. The Harrison's approach is based on the following set of equations:

$$
\begin{array}{r}
\frac{\partial \rho}{\partial t}+\vec{\nabla}_{r} \cdot\left(\rho+\frac{P_{t h}}{c^{2}}\right) \vec{u}=0 \\
\frac{d \vec{u}}{d t}=-\vec{\nabla}_{r} \phi \\
\nabla_{r}^{2} \phi=4 \pi G\left(\rho+\frac{3 P_{t h}}{c^{2}}\right),
\end{array}
$$

which are respectively the continuity equation, Poisson's equation and the motion equation. Posteriorly, Harrison [4] derived the motion equation by introducing a non-uniform pressure, namely:

$$
\frac{\partial \vec{u}}{\partial t}+\vec{u} \cdot \vec{\nabla}_{r} \vec{u}=-\vec{\nabla}_{r} \phi-\left(\rho+P_{t h}\right)^{-1} \nabla_{r} P_{t h} .
$$

In the above equations $\rho, P_{t h}, u$ and $\phi$ denote respectively the density of matter, the thermodynamic pressure, the velocity field and the gravitational potential.

The field equations for a homogeneous and isotropic universe are obtained by using equations (1), (2) and
(3). These equations are identical to the ones obtained in the framework of general relativity in the comoving coordinate system.

However, it was observed in the end of the appendix II of the R.K.Sachs and A.M Wolfe work that the perturbed modified field equations to the first order are not the same in the general relativity and Newtonian cosmology. Therefore, the density contrast equation for both theories are quite different.

This ambiguity was solved by Lima, Zanchin and Brandenberger [6] that derived the continuity equation in the modified Newtonian approach to cosmology when pressure effects are included. The new continuity equation is completely consistent with relativistic perturbation theory and solves the contradiction pointed out by Sachs and Wolfe.

In this paper, we generalize the evolution equation for the density contrast obtained by Lima et. al., for the universe with particle production and obtained the growing and decaying modes for the density contrast.

In our work we introduce a variable $(\xi)$ that depends of the age of the universe and of the growing mode of the density contrast. The use of $\xi$ can discern the cosmological model that furnishes a better concordance with the experimental evidence about the age of the universe and the time of structure formation.

This paper is organized as follows: we obtain in section II, in the linear approximation, the time evolution equation for the mass density contrast for a Newtonian universe with matter creation. In section III we derive 
the time dependence of the density contrast in which the source of particle production is $\Psi=3 n \beta H$. We obtain a range for the parameter $\beta$ that updates the range obtained in the literature when the question of the age of the universe is involved. Finally we use the reach of the non linear regime to establish a criterion about the most adequate cosmological scenario.

\section{Equation for the density con- trast}

In the context of matter creation the thermodyamical conservation law reads:

$$
\frac{d \rho}{d V}+\frac{1}{V}\left(P_{t h}+\frac{\rho}{c^{2}}\right)+\frac{h}{n V} \frac{d}{d V}(n V)=0,
$$

where $n$ is the particle number density, $h=\rho+P_{t h}$ is the enthalpy per unity volume. Consequently the continuity equation becomes

$$
\frac{\partial \rho}{\partial t}+\nabla_{r} \cdot(\rho \vec{u})+\frac{P_{t h}}{c^{2}} \nabla_{r} \cdot \vec{u}=\frac{h}{n} \Psi
$$

where $\Psi$ is the source of particle production. The other hydro-dynamical equations that describe the cosmic fluid are the momentum conservation equation and Poisson's equation, respectively:

$$
\frac{\partial \vec{u}}{\partial t}+\vec{u} \nabla_{r} \vec{u}=-\nabla_{r} \Phi-\left(\rho+\frac{P}{c^{2}}\right)^{-1} \nabla_{r} P
$$

and

$$
\nabla_{r}^{2} \Phi=4 \pi G\left(\rho+\frac{3 P}{c^{2}}\right)
$$

$\Phi$ is the gravitational potential and $P$ is the total pressure that includes the thermodynamical pressure and the creation pressure $\tilde{P}$, so

$$
P=\tilde{P}+P_{t h}
$$

The creation process will be considered adiabatic, consequently the pressure creation is given by [7] :

$$
\tilde{P}=-\frac{h \Psi}{n \theta} .
$$

In order to obtain a cosmological scenario with matter creation one still needs to provide the state equation

$$
P_{t h}=\nu \rho \text {. }
$$

Using the field equations for FRW model [8] with particle production and the state equation above, we can write a differential equation for the scale factor:

$$
R \ddot{R}+\left(\frac{3 \nu+1}{2}-\frac{(\nu+1) \Psi}{2 n H}\right)\left(\dot{R}^{2}+\kappa\right)=0
$$

Our main aim in this section is to find the differential equation that governs the evolution of the density contrast in a universe where the particle production is given by $\Psi=3 n \beta H$, where $\beta$ is a constant. Note that, put $\beta=0$ in equation (11) implies $\Psi=0$ and we recover the standard Friedmann model.

To study the evolution of small fluctuations in an expanding universe we consider the standard perturbation ansatz:

$$
\begin{gathered}
\rho=\rho_{b}(t)\{1+\delta(r, t)\}, \\
P_{t h}=P_{t h b}(t)+\delta P_{t h}(r, t), \\
\Phi=\Phi_{b}(r, t)+\varphi(r, t), \\
\vec{u}=\vec{u}_{b}(t)+\vec{v}(r, t), \\
\tilde{P}=\tilde{P}_{b}(t)+\delta \tilde{P}(r, t) .
\end{gathered}
$$

The quantities carrying the subscript $b$ represent the homogeneous solution to the unperturbed equations. Inserting the above expressions into eqs. (5), (6) and (7) we get to first order in perturbations

$$
\begin{gathered}
\dot{v}+\frac{\dot{R}}{R} v=\frac{-1}{R} \nabla \varphi-\frac{\nu-\beta(\nu+1)}{(\nu+1)(1-\beta)} \frac{\nabla \delta}{R}, \\
\nabla^{2} \varphi=4 \pi G \rho_{b}(t) R^{2} \delta\{1+3 \nu-3(\nu+1) \beta\}, \\
\dot{\delta}=\frac{\nu+1}{R} \nabla v(1-\beta) .
\end{gathered}
$$

Change to comoving coordinates following standard lines [9], eliminating the peculiar velocity from equations (17) and (19) and using (18), is ready that

$$
\begin{aligned}
& \ddot{\delta} \quad+2 \frac{\dot{R}}{R} \dot{\delta}+4 \pi G \rho_{b}(t) \delta\{1+3 \nu-3(\nu+1) \beta\} \\
& =\quad(\nu+1)(\beta-1)(\nu-\beta(\nu+1)) \frac{\nabla^{2} \delta}{R^{2}} .
\end{aligned}
$$

This is the differential equation that governs the evolution of density contrast in the presence of matter creation, when we describe a matter distribution with uniform pressure using the modified Newtonian equations. It is spatially homogeneous, so we expect to find plane wave solutions. The difficulty to apply Newtonian cosmology in the study of scalar perturbations is related with the impossibility of the Newtonian scenario to describe long wavelengths perturbations. The cosmological perturbations can be of the two kind according to the wavelength $\lambda$, so that $\lambda>d_{H}$ or $\lambda<d_{H}$, where $d_{h}$ is the Hubble sphere. Perturbations with wavelengths larger than Hubble scale requires some form of a general relativistic theory of perturbations. For wavelengths smaller than Hubble scale the evolution of mass density can be studied using Newtonian theory. Application of Newtonian equations is further restricted 
to non-relativistic matter and cannot be used for relativistic components even for scales smaller than Hubble radius.

The reexamined Newtonian equations indicate a way of obtaining the same evolution equation for the density contrast as could be by the full relativistic approach. In this way one can extend the domain of validity of equation (20) in order to analyze perturbations even in the radiation dominated phase and to apply the large wavelength limit.

Next, we derive the solution of the density contrast when the source of particle production is $\Psi=3 n \beta H$, concentrating our attention on the growing mode. The principal advantage of this approach is the relative facility to obtain the perturbed equation for the density contrast which is the same equation as in general relativity when $\beta=0$ in the synchronous and comoving coordinates.

Although the gauge invariant approach is conceptually more attractive since there is no need to identify the physical and unrealistic space-times, nevertheless it is more complicated and the physical meaning of gauge independent variables do not in general possess any simple interpretation. Besides, the gauge mode that emerges from the differential equation for the density contrast is generally related with some kind of decaying mode when the universe is homogeneous and isotropic [10].

\section{The growing mode and the age of the universe}

If the space time has uniform curvature, the line element is given by

$d s^{2}=d t^{2}-R^{2}(t)\left(\frac{d r^{2}}{1-\kappa r^{2}}+r^{2} d \theta^{2}+r^{2} \sin ^{2}(\theta) d \phi^{2}\right)$.

The evolution of the scale factor is obtained from solutions of differential equation [8]

$$
R \ddot{R}+\Delta\left(\kappa+\dot{R^{2}}\right)=0,
$$

where

$$
\Delta=\frac{3}{2}(\nu+1)(1-\beta)-1
$$

Explicit solutions for differential equation (22) when $\kappa= \pm 1$ for any value of $\beta$ are not easy to obtain. Besides, $\beta<0$ broken the second law of thermodynamics and $\beta>1$ broken the dominant energy condition. We have managed to find a class of exact solutions for the following cases:

$$
\kappa=0 \beta \neq 1 \longrightarrow R=R_{0}\left(\frac{t}{t_{0}}\right)^{\frac{1}{\Delta+1}},
$$

$$
\begin{gathered}
\kappa=0 \beta=1 \longrightarrow R=R_{0} e^{\frac{t}{t_{0}}}, \\
\kappa= \pm 1 \beta=1 \longrightarrow R=\frac{1}{2}\left\{\frac{\kappa+e^{ \pm 2 C_{1}^{\frac{1}{2}}}\left(t+C_{2}\right)}{e^{ \pm C_{1}^{\frac{1}{2}}}\left(t+C_{2}\right) C_{1}^{\frac{1}{2}}}\right\} .
\end{gathered}
$$

The subscript 0 alludes to the present time and $C_{1}$ and $C_{2}$ are integration constants.

Whether the universe turns out to be spatially closed, open or precisely flat remains an empirical question. The recent results of the Boomerang provide convincing evidence in favor of the standard paradigm: The universe is flat, however the possibility that the universe might be spatially closed do not be discarded [11]. Consequently, we consider the universe as flat and $\beta \neq 1$ for subsequent calculation.

Using the scale factor (24) then the mass-energy density is given by:

$$
8 \pi G \rho_{b}=\frac{1}{(\Delta+1)^{2} t^{2}} .
$$

Using equations (27), (24) and the long wavelength limit into equation (20) we finally obtain

$$
\ddot{\delta}+\frac{\alpha}{t} \dot{\delta}+(\alpha-2) \frac{\delta}{t^{2}}=0
$$

where

$$
\alpha=\frac{4}{3(\nu+1)(1-\beta)} .
$$

Solutions for equation (28) are

$$
\begin{array}{r}
\delta_{-}=C_{1} t^{-1}, \\
\delta_{+}=C_{2} t^{2-\frac{4}{3(1-\beta)(\nu+1)}} .
\end{array}
$$

In the absence of particle production, $\beta=0$, relations (30) and (31) become the usual density contrast for Friedmann models without creation [12]. Although the decreasing mode can be important in some circumstances, the increasing mode is responsible for the formation of cosmic structures in the gravitational instability picture. Taking into account the decreasing mode the universe would not have been homogeneous in the past. Besides, Peebles argued that a growing mode that starts to grow just after the end of radiation era has a negligible component of decaying solution [13].

One of the problems of the standard FRW model is related to the age of the universe; it is younger than it should be according to the experimental values. However, in the framework of particle production the value of the age of the universe is more compatible with the 
experimental evidences. To show this we define the quotient $\zeta$ given by:

$$
\zeta=\frac{H_{c}}{H_{F}}
$$

at the same time coordinate. The subscript $\mathrm{F}$ and $\mathrm{C}$ refer respectively to the Friedmann standard model and the model with particle creation.

Considering the scale factor (24) one obtains

$$
\zeta=\frac{1}{1-\beta} .
$$

If we expect that the open system model furnishes an oldest universe, then $\zeta>1$ implying $0<\beta<1$. Using the oldest globular clusters, B. Chaboyer [14] infers a range for the age of the universe, namely 9.6Gyr $<t_{\text {universe }}<15.4 G y r$. Consequently, the Hubble constant for the standard model lies in the interval

$$
43.3<H_{0}<69.4 \text {. }
$$

This range does not agree with the interval for $H_{0}$ estimated recently by Willick and Batra [15], namely:

$$
80<H_{0}<90 \text {. }
$$

One can easily verify that there is a small overlap between (34) and the values obtained by Richtler and Drenkhahn [16], given by

$$
68<H_{0}<76 \text {. }
$$

Using the scale factor (24) for dust matter universe, the Hubble function for a universe with particle production is given by

$$
H=\frac{2}{3(1-\beta) t} .
$$

We can conciliate the ranges (35) and (36) using the Hubble function above, where the creation parameter $\beta$ lies in the interval

$$
0.13<\beta<0.52 \text {. }
$$

This result updates the interval for $\beta$ determined by Lima et al [8] $(0.34<\beta<0.60)$.

Now, we study the reach of non linear regime, identifying the epoch for the reach of the non linear regime with the epoch for the formation of the super clusters. In addition, we suppose that the star formation is posterior to the formation of the super clusters. First, because the density of the super cluster in the universe is approximately equal to the universe density. Second, considering the universe to form "from top down" is acceptable for some type of models, for example the hot dark matter model [17]. In other words, we want to infer that the star formation is posterior to the super cluster formation and, consequently, to the reach of the non linear regime. The growing mode for the density contrast for the FRW standard model can be written as

$$
\delta_{+}=\delta_{d}\left\{\frac{t}{t_{d}}\right\}^{2 / 3},
$$

where the subscript $d$ refers to decoupling time. Typically we have $\left(\frac{\delta \rho}{\rho}\right)_{d e c}<\left(10^{-2}-10^{-3}\right)$ [17], for a decoupling time of the order $10^{5} \mathrm{ys}$. Substituting the decoupling time and the corresponding anisotropy, the reach for the non linear regime for FRW standard model occurs in the range $10^{-1}$ Gyr $<t<3.16 \times$ Gyr. Note that, the standard model furnishes a sub estimate age for the universe, around 9 Gyr. Now, if we consider the difference between the age of the universe and the reach of the non-linear regime we obtain the interval $5.8 G y r<t<8.9$ Gyr for the age of the super cluster. In the literature it is known that the age for the oldest stars in our galaxy is around 12 Gyr [14].

The open system cosmology furnishes a reasonable solutions for the age of the universe. What happens with the reach of the non linear regime for the density contrast?

To solve this questions we introduce a variable $\xi$ that relates the age of the universe and the growing mode for the density contrast in order to discern among more suitable cosmological model, it is given by:

$$
\xi=\frac{\delta_{+c}}{\delta_{+F}} \zeta .
$$

A cosmological model with better characteristics to represent the universe must be satisfied if $\xi>1$. Taking into account the growing mode (31) and relation (33), the condition (40) implies that $\beta<0$. Negative values for the parameter $\beta$ violates the second law of thermodynamics. Then we conclude that the respective cosmological model to the source given by $\Psi=3 n \beta H$ does not give a better result than the usual FRW model when the age problem and the reach of the non-linear regime are taking together. On the another hand if we examine the difference of the age for the universe and the reach for the non linear regime in the context of the open system cosmology with a particle source given by $\Psi=3 n \beta H$, we note that this difference is smallest than the result obtained in the FRW framework.

\section{References}

[1] E.A. Milne, Quart. J. Math 5, 64 (1934).

[2] W.H. Mc Crea and E.A. Milne, Quart. J. Math 5, 73 (1934).

[3] E.R. Harrison, Annals of Physics 35, 437 (1965).

[4] E.R. Harrison, Reviews of Modern Physics 39, 862 (1967). 
[5] R.K. Sachs and A.M. Wolfe, ApJ 1, 73 (1967).

[6] J.A.S. Lima, V. Zanchin and R. Brandenberger, MNRAS 291, L1 (1997).

[7] M.O. Calvão, J.A.S. Lima and I. Waga, Phys.Lett. A 162, 223 (1992)

[8] J.A.S. Lima, A.S.M. Germano and L.R.W. Abramo, Phys. Rev. D53, 4287 (1996).

[9] P.J.E. Peebles, The Large Scale Structure of the Universe, (Princeton University Press, Princeton, 1980).

[10] J. Hwang and E.T. Vishniac, ApJ 353, 1 (1990).

[11] Martin White, Douglas Scott and Elena Pierpaoli, astro-ph/0004385.
[12] S. Weinberg, Gravitation and Cosmology: Principles and Applications of the General Theory of Relativity, (Jonh Wiley \& Sons, New York, 1972).

[13] J. Peebles et al., ApJ 147, 859 (1967).

[14] Brian Chaboyer, ApJ 494, 96 (1998).

[15] J.A. Willick and P. Batra, astro-ph/0005112.

[16] Tom Richtler and Georg Drenkhahn, astro-ph/9909117.

[17] E.W. Kolb and M.S. Turner, The Early Universe, (Addison-Wesley, Redwood City, California, 1990). 